\title{
Mouse Avatars of Human Cancers: The Temporality of Translation in Precision Oncology
}

\author{
Sara Green ${ }^{1,2}$, Mie S. Dam² \& Mette N.l Svendsen ${ }^{2}$ \\ ${ }^{1}$ Section for History and Philosophy of Science, Department of Science Education, University of \\ Copenhagen, Niels Bohr Building (NBB), Universitetsparken 5, 2100 Copenhagen Ø, DK. Email: \\ sara.green@ind.ku.dk \\ ${ }^{2}$ Centre for Medical Science and Technology Studies, Department of Public Health, University of \\ Copenhagen, DK.
}

\begin{abstract}
Patient-derived xenografts (PDXs) are currently promoted as new translational models in precision oncology. PDXs are immunodeficient mice with human tumors that are used as surrogate models to represent specific types of cancer. By accounting for the genetic heterogeneity of cancer tumors, PDXs are hoped to provide more clinically relevant results in preclinical research. Further, in the function of so-called "mouse avatars", PDXs are hoped to allow for patient-specific drug testing in real-time (in parallel to treatment of the corresponding cancer patient). This paper examines the circulation of knowledge and bodily material across the species boundary of human and personalized mouse model, historically as well as in contemporary practices. PDXs raise interesting questions about the relation between animal model and human patient, and about the capacity of hybrid or interspecies models to close existing translational gaps. We highlight that the translational potential of PDXs not only depends on representational matching of model and target, but also on temporal alignment between model development and practical uses. Aside from the importance of ensuring temporal stability of human tumors in a murine body, the mouse avatar concept rests on the possibility of aligning the temporal horizons of the clinic and the lab. We examine strategies to address temporal challenges, including cryopreservation and biobanking, as well as attempts to speed up translation through modification and use of faster developing organisms. We discuss how featured model virtues change with precision oncology, and contend that temporality is a model feature that deserves more philosophical attention.
\end{abstract}

Keywords: Precision oncology; Personalized medicine; PDXs; Translational models; Surrogate models; Hybrid models 


\section{Introduction}

The laboratory mouse model has historically been essential in cancer research, and is still widely used also for drug development and drug testing (Rader 2004; Lewis et al. 2012). Discrepancies between the physiology of human and non-human organisms have, however, also led to critical examination of the use of mice and other non-human organisms as translational models in cancer research and beyond (Shanks et al. 2009; Denayer et al. 2014; Leenars et al. 2019). An important strategy to overcome this translational gap is to develop hybrid models, such as mouse xenografts (from Greek, xenos, foreign) in which human tissues are growing inside a mouse. In this paper we focus on patient-derived xenografts (PDXs) in precision oncoloy, which have co-evolved with the increasing focus on molecular alterations in cancer classification and choice of targeted treatments (Day et al. 2015). PDXs are hoped to better account for how "each individual cancer is characterized by patient-specific molecular events" (Izumchenko et al. 2017, p. 2595), and thereby to improve the clinical translation from animal model to human patient.

Patient-derived models are philosophically intriguing because they stretch the concept of an animal model, which is typically taken to even out the heterogeneity of human bodies. In the words of Harrington:

[T] he animal is said by scientists to "stand in" for the human, to "simplify" the process of knowledge attainment. Reasons for using the animal encompass the ethical - that it is not considered moral or just to experiment on humans - and heterogeneity, with every human body being different, nonstandardized in genetic and physiological detail. This is in contrast to the way in which the honed and standardized animal model is framed; in the case of the mouse, the model being described as "pure biological reagents" (Harrington 2013, p. 186, our emphasis)

Rodents often work as standardized models in the way described by Harrington. For instance, mice are often used as model organisms in community-wide studies of the shared genetic and phenotypic similarity between mice, humans, and other organisms (Leonelli and Ankeny 2012). Model organisms are characterized by a broad representational scope and a high degree of standardization, which is achieved not only through phylogenetic homologies but also through experimental manipulation, coordinated experimental protocols, and data infrastructures (Ankeny and Leonelli 
2011). PDXs notably differ from the use of rodents as model organisms in aiming to represent specific, rather than general, targets.

Bolker's (2009) distinction between exemplary models and surrogate models is useful to clarify this difference. Model organisms are exemplary models in the sense that selected features make up an exemplar for the study of traits that are shared by a large class of organisms. In contrast, the aim of surrogate models is to substitute specific targets, such as specific human patients in clinical trials. As substitutes for patients, they are often used for diagnostic purposes, e.g., for testing of the efficacy of treatments (Bolker 2009, p. 488). Surrogate models thus combine two functions of the use of animals in biomedical contexts: the representation of a human disease and the use of animals as diagnostic tools. ${ }^{1} \mathrm{We}$ contend that PDXs can be considered as extreme types of surrogate models, since the de facto similarity of the human tumor and transplanted tumor tissue is hoped to ensure that the surrogate responds to an intervention (cancer treatment) in the same way as would the specific patient. Thus, accurate representation of selected characteristics of specific patients is key to their role as diagnostic tools. But, as we shall see below, diagnostic purposes can also conflict with the possibility of representing the complexity of human disease in an animal model.

Per design, PDXs are intended to substitute human patients, a feature which is highlighted in both public media and scientific papers. PDXs have been viewed as "the closest model to human cancer available without using humans themselves" (Scudellari 2015), and hence as more direct "stand-ins for real people" (Perry 2013). Such descriptions seem to blur the very distinction between experimental animal model and human targets, forming a hybrid entity at the interface of species boundaries. Similarly, the notion of a mouse avatar highlights how PDXs are personalized models that are envisioned to allow "each patient to have their own tumor growing in an in vivo system" (Malaney et al. 2014, p. 1). In this function as a patient-specific in vivo model, drug testing is not only an intervention on an experimental system or animal model, but also on a part of the individual patient (Boniolo 2017, p. 29). As a result, it is hoped that results from PDXs can be more easily extrapolated to the human context and offer better guidance in clinical decision making. Yet,

\footnotetext{
${ }^{1}$ Some animals function as diagnostic tools without representing the human disease or medical condition of interest. An interesting historical example is the use of mice and rabbits for pregnancy testing and detection of malignant tumors in the late 1920 and 1930s (Olszynko-Gryn 2014). Injection of human urine into these organisms, and subsequent observation of physiological changes (although dissimilar to the human condition), served as a test for hormonal alterations related to pregnancy and cancer in humans. We thank Rob Kirk for bringing this example to our attention.
} 
the interspecies intimacy achieved through xenografting of human tissues does not automatically ease medical translation.

In the following, we analyze the potential and challenges associated with attempts to shorten translational paths between experimental models and human targets by growing patient-derived tumors in mice. We are interested in how model development materializes in concrete practices in precision oncology. This involves not only theoretical considerations of representational relations, but also an analysis of practical constraints that influence attempts to close translational gaps between mouse models and human patients. For this purpose, we combine a philosophical analysis of position papers and scientific publications on PDXs with interviews and interactions with cancer researchers working with murine models (standardized as well as PDXs). Moreover, we further supplement the analysis with ethnographic fieldwork in a translational project aiming to conduct patient-specific drug screens based on PDXs and patient-derived tumor organoids. By drawing on this material, we unpack factors that challenge and sometimes hinder material transfer and clinical translation through the use of PDXs, as well as strategies to address the challenges.

In line with other papers in this topical collection, we are inspired by Netz's (2004) historical analysis of how modernity developed through restrictions on the spatial freedom of animals and humans, of keeping out as well as keeping in. But whereas the barbed wire primarily symbolizes the control of the movement of bodies in space, we place further emphasis on the potential to facilitate and control movement of bodies in time. It is well-known that temporal constraints are important for organism choice in biological research, e.g., in consideration of the length of the life cycle of candidate organisms to study embryonic development, toxicology testing, reproduction, or aging (Dietrich et al. 2019). So far, however, less attention has been given in the philosophical literature to how the temporal characteristics of data and models affect their status as evidence for practical purposes (see however Leonelli 2018). We document how the temporality of PDXs plays out in two different contexts: in practices that aim to construct in vivo models for realtime clinical drug testing (Section 3.1) and as pre-clinical research models (see Section 3.2). The temporal challenge is more pressing in the former context due to the importance of aligning model development to the time window of clinical decision making (see also Svendsen et al. 2018). In both contexts, however, cryopreservation and biobanking are central procedures for managing scarcity and instability of living tumor models (Section 3.3).

We begin with an introduction to the historical background for PDXs (Section 2), and then explore how challenges described in historical papers play out in the contemporary context of 
precision oncology (Section 3). We then reflect upon potential future directions for the field, including attempts to make cancer models more or less "humanized", in some cases by trading representational accuracy for features that speed up the process of xenograft development (Section

4). We end with concluding remarks on how ambitions for patient-specific drug screening influence featured virtues of translational models (Section 5).

\section{Mouse models and PDXs in a historical perspective}

Differences between human and mice constitute both enabling and limiting constraints for translational research and practice. On the one hand, mice are considered sufficiently morally different from humans to allow for experimental interventions that would not be ethically justifiable on human subjects (Svendsen and Koch 2013). On the other hand, physiological similarities of mice and human biology are required for mice to work as stand-ins or proxies for aspects of human diseases (Lewis et al. 2012, p. 778). As expressed in the following quote from an interview with a cancer researcher in molecular medicine, animal models are central not only for drug approval but also for publication:

In my field, animal research is essential - it's hard to publish anything in good journals if you cannot show results in some kind of animal experiment. At least if you like us do translational research and study the effects of treatment on cancer cells. It's no longer enough to show it in a cell culture in vitro - you have to be able to demonstrate it in an animal model.

Whereas in vitro models are easier to handle in the lab, the mouse model is often seen as superior because of the presence of the in vivo metabolism and spatial structure of the cancer tissue. On the other hand, the differences between the biology of mice and humans have been problematized as a complicating factor for translational inferences (van der Worp et al. 2010; Denayer et al. 2014). Yet, the possibility of extrapolating from experimental organisms to human targets is not completely fixed by the presence or absence of a given trait-specific phylogenetic homology, but is sufficiently plastic to be experimentally engineered (Maugeri and Blasimme 2011). The strategy to grow human tumors in immunocompromised murine models illustrates this well. 
This is a preprint of an article published in History and Philosophy of the Life Sciences. The final authenticated version is available online at: https://doi.org/10.1007/s40656-021-00383-w

Mouse xenografts are becoming increasingly common in preclinical cancer studies ${ }^{2}$, and have recently received renewed attention with the call for more personalized (PDX) models in precision oncology. Yet, it is important to note that the exploration of mouse xenografts as patientderived models has a long history (see e.g., Ovejera et al. 1978; Houchens et al. 1983; Taetle et al. 1986; Winograd et al. 1988). ${ }^{3}$ Early papers report on successful matching of treatment outcomes in xenograft systems and human patients, but also on difficulties of aligning engraftment rates with the narrow time-window of clinical decision making. As we shall document below, a historical example illustrates challenges that are also of relevance for the contemporary context of precision oncology.

Heinz-Herbert Fiebig from the University of Freiburg was one of the pioneers in the development of PDXs. Already in the 1980s, Fiebig and colleagues conducted hundreds of successful tumor xenotransplantations and comparisons of treatment effects in patients and xenograft mice (Fiebig et al. 1984; 1985). The protocol for development of tumor xenograft models relied on a procedure called in vivo passaging, which is also used today (see Section 3.2). Passaging refers to a process in which tumor samples from humans grow up to a certain size in a mouse host, the tumor (or a fragment of the tumor) is then removed from the mouse host and split into smaller pieces, which are grown in new mouse models. In this way, the amount of human tumor material can be expanded through several hybrid models representing the same patient's tumor. In the studies of Fiebig and colleagues, most tumors fragments were obtained from surgeries of the primary tumors and transplanted subcutaneously (under the skin of the dorsal region) into the mouse host. ${ }^{4}$ Therapeutic experiments were conducted in animals with two tumor implants, and treatment effects were evaluated in comparison to untreated mice controls, as well as to treatment effects in the human patients (Fiebig et al. 1984).

Fiebig (1988) reports on the results of 406 successful tumor xenotransplantations, which gave rise to 80 comparisons of test results from tumor xenografts and patients. The tumor xenograft test results were found to correctly predict treatment resistance in $96 \%$ of the cases, and to predict

\footnotetext{
${ }^{2}$ A recent review in Nature found that $89 \%$ of all preclinical studies in oncology published in 2016 involved mouse xenografts (Gengenbacker et al. 2017). The vast majority of these are based on standardized human cancer cell lines. 3 The early models were "nude mice", so called because a genetic mutation disruptive of T-cell development also made the mice hairless (Flanagan 1966; Pantelouris 1968; Rygaard and Poulsen 1969). Another common xenograft model used today are so-called SCID mice, that have an immune deficiency effecting B and T lymphocyte development without making them hairless.

${ }^{4}$ Fiebig et al. (1984) describes how 4 initial tumor fragments of $5 \times 5 \times 0.5-1 \mathrm{~mm}$ in diameter 4 were injected into the first "generation" of mice. When the tumor reached a diameter of 1-1.5 cm, it was studied histologically, split up, and grown in other mice. Importantly, this amount of starting material differs considerably from the clinical contexts we examine in Section 3.1, where practitioners have to rely on tumor samples from a few needle biopsies.
} 
treatment response in $90 \%$ of the cases. Given the difficulty of predicting treatment response in cancer patient in general, this must be seen as a rather extraordinary and successful result. Nevertheless, Fiebig repeatedly stresses the difficulties of developing the model systems within a clinically meaningful time-window, as seen in the following passage from the paper abstract (a similar passage can be found in the conclusion):

Despite great efforts to obtain a large number of comparisons, only 32 test results were available before the patients needed treatment. Therefore, our study has shown that the xenograft system will not have practical significance in determining individual patient treatment. (Fiebig 1988, p. 25)

The quote illustrates how the xenograft system is not only evaluated on its representational accuracy and predictive capacity, but also by practical considerations. Particularly important is the possibility of aligning model development to the temporality of the clinic.

To obtain enough tumor material and ensure stable growth of the tumors in the xenografts, Fiebig and colleagues found it necessary to conduct several passages. Moreover, experimental testing of treatment effects had to be done between passage 2 and 6 where tumor growth and histological features were found to be most regular, which further delayed drug testing (Fiebig et al. 1984). Only 50\% of the implanted tumors were possible to grow in and passage between mice, and even in successful cases 4-8 months were needed to obtain a result. Fiebig and many others saw the time-lag as a fundamental limit to the clinical relevance of the xenograft system. In addition to temporal alignment, Fiebig et al. mention the expenses of special laboratory requirements, such as laminar flow cabinets to avoid infections in immunocompromised mice. However, the authors highlight the benefits of investing in xenograft systems as preclinical models in research on cancer drug development and drug testing (Fiebig et al. 1984; Fiebig 1988; see also Schulz et al. 1990). In the following, we explore how the temporal challenge plays out in the contemporary context of precision oncology.

\section{PDXs in contemporary practice}

\section{1. "Mouse avatars" for patient-specific drug screening}

Patient-derived xenografts have recently had a revival in the context of precision medicine. In this context, the genetic heterogeneity of human cancers has been highlighted as a feature that, at least 
This is a preprint of an article published in History and Philosophy of the Life Sciences. The final authenticated version is available online at: $\mathrm{https}$ ://doi.org/10.1007/s40656-021-00383-w

partly, explains the discrepancy between drug testing on standardized animal models and individual patients (Xu et al. 2019). Individualized mouse models have been promoted as a solution because they, though at higher costs, can account for the heterogeneity of cancer tumors found in the clinic. A number of recent publications use the term "mouse avatar", a concept illustrated in Figure 1 (from Hidalgo et al. 2014). The figure shows how tumor fragments from a patient are transplanted into an immunocompromised mouse, from which several PDXs are developed through passaging procedures similar to those in Fiebig's studies. In addition, the figure illustrates how PDXs today are used in combination with modern technologies, such as genomic profiling, which are used to identify potential biomarkers and targeted drugs via mining of genomic-drug databases. Mouse avatars are here considered as a tool for patient-specific drug screening, i.e., as ways to find the most effective treatment option for a specific patient. ${ }^{5}$

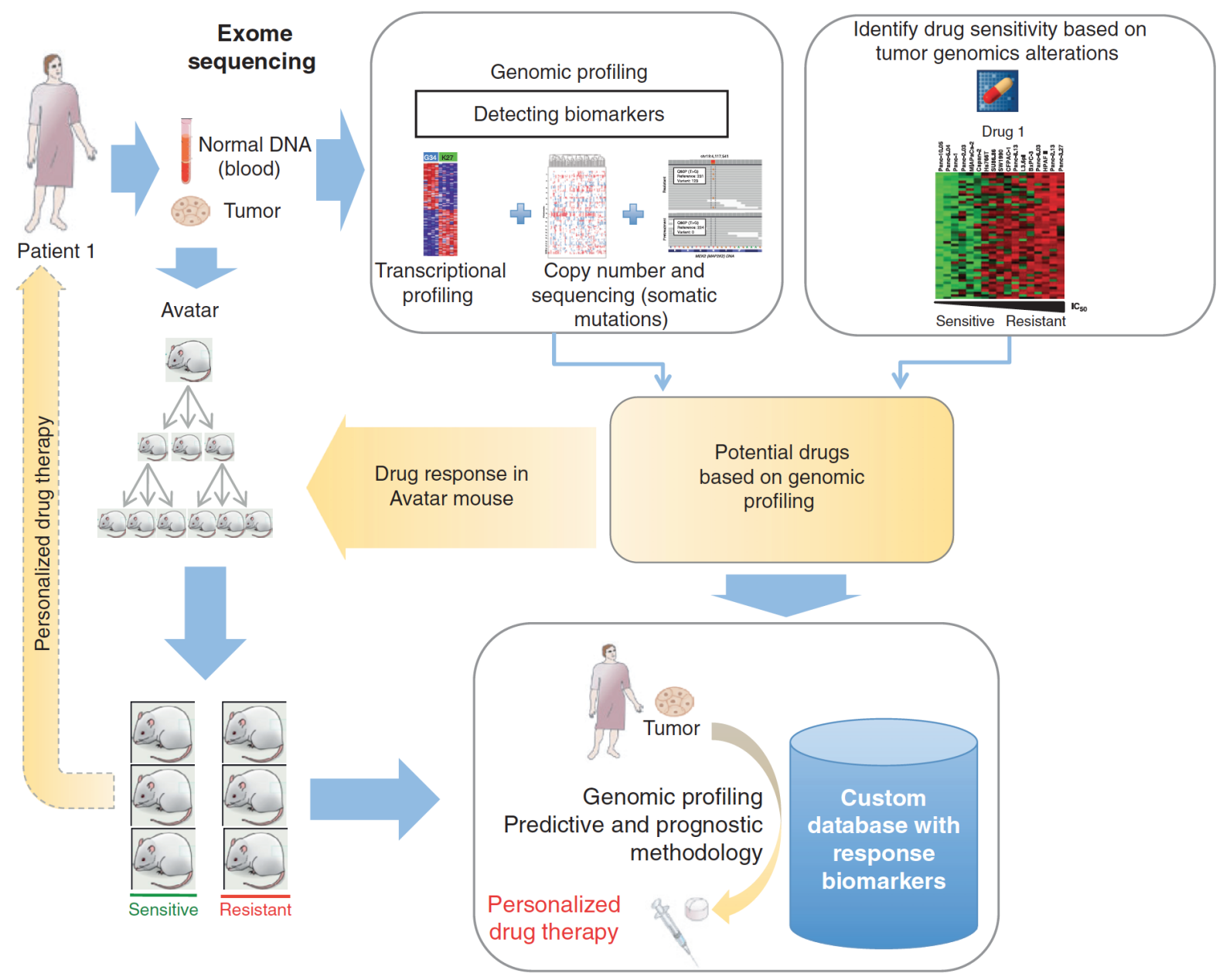

\footnotetext{
${ }^{5}$ In the words of Hidalgo, "the Avatar model can be used to test and rank [...] potential treatments to be administered to the patient" (Hidalgo et al. 2014 p. 1007).
} 
Figure 1. Illustration of the concept of a mouse avatar. Reprinted from (Hidalgo et al. 2014) with permission from AACR.

Review articles differ in their framing of the potential of PDXs as mouse avatars of individual patients. Some emphasize that mouse avatars allow for "a quick assessment of the safety and efficacy profiles of an investigational drug or novel drug combinations" (Malaney et al. 2014, p. 9). Indeed, diagrams like the one in Figure 1 give the impression that PDXs are an almost inexhaustible testing resource and that clinical treatment and laboratory drug testing can be run in "real time". However, the paper from which we borrow the figure also highlights that "in most patients the approach is not feasible for reasons such as failure of the tumor to engraft, lack of effective agents, and length of time required for a complete study" (Hidalgo et al. 2014, p. 1007). The phrase "the right drug for the right patient at the right time" has become a kind of slogan for precision oncology. For most cancer patients, however, targeted drugs for their specific cancers are currently unavailable (Hey and Barsanti-Innes 2016; Plutynski, forthcoming). The discussion on PDXs further shows that even when there are drugs and screening tools available, the right drug may not be identified at the right time.

As part of an ethnographic research project, we have followed a translational project aiming to realize the vision of patient-derived in vitro and in vivo models (PDXs) for patient-specific drug screening. The project is a close collaboration between an experimental lab and a Phase I oncology clinic. Patients involved in the project are metastatic cancer patients that have not responded, or do no longer respond, to standard treatment, and the hope is that new experimental treatments could benefit this patient group. Instead of exposing the patient directly to an off-label drug ${ }^{6}$ based on the molecular profile of the tumor, the aim of the project was to first screen for the efficacy of the targeted drugs on tumor organoids ${ }^{7}$ and PDXs based on cells from the same patient. The project application contained a figure similar to Figure 1. The figure illustrates the ideal diagnostic pipeline, where biopsy samples from metastatic cancer patients are used for both genomic analysis (to identify potential targeted drugs), and for development of personalized drug testing models. In the research application, PDX models were presented as playing a central role in allowing for in

\footnotetext{
${ }^{6}$ Off-label means that an approved medication is being used in a manner not specified in the FDA's approved packaging label. An example could be the use of a drug approved for treatment of colon cancer on breast cancer patients, if the patients share the same genetic variants (in this context, the treatment becomes experimental although the drug is FDA approved).

${ }^{7}$ Tumor organoids are 3D cultures of tumor cells, which grow in a matrix hydrogel that mimics some aspects of the tumor micro environment.
} 
vivo drug screening, parallel to the drug screen of tumor organoids in vitro. But the envisioned translational path was hampered by practical limitations similar to those reported by Fiebig.

A post-doctoral researcher in the project explained that organoids and PDXs are viewed as more biologically realistic models than traditional 2D in vitro cultures, because they better recapitulate the genetic heterogeneity and spatial structure of human tumors (see also Green et al. forthcoming). On the other hand, she also pointed to the limitations arising when you have to grow human cancers in immunocompromised mouse models that are dissimilar to human patients in many ways. The greatest challenge in this project is, however, the discrepancy between growth rates of patient-derived models and the time-window for making treatment decisions in the clinic. When asked about the status of PDX models in the project, the post-doctoral researcher replied:

As it has been until now, considering the need to treat some patients fast, it has not been something that we have time to do. It has also been a question of whether we have enough [tumor] material to do what we have to do. And to freeze the samples and all that, before even considering how to set up the PDX. What material do we have left to put in the mouse?

Since the patients in this context most often have inoperable metastatic cancer, the amount of tumor material is limited to a few needle biopsies. The struggle to align the temporality of the laboratory and clinic is illustrated in the following quote by the same post-docoral researcher, in response to information that a patient involved in the trial has died before the test results could be used to offer a new treatment:

Oh. That is so sad! You see, it has been like that with a lot of [the organoids] I've had. When I've done the drug screen and sent it to the clinic, half of the patients [that I have made drug screens for] have died. Then I'm left thinking: could something have been done if I had been faster?

Ethical constraints on the access to experimental cancer patients, as well as to the number of justifiable biopsies, aggravate the temporal challenge. Although the experimental protocol is only open for incurable cancer patients with good performance status, ${ }^{8}$ the time-window for clinical

\footnotetext{
${ }^{8}$ Performance status refers to a numerical score (ECOG) that reflects a patient's ability to function in daily life. To be included in the experimental treatment protocols we have examined in Denmark, patients must have exhausted
} 
This is a preprint of an article published in History and Philosophy of the Life Sciences. The final authenticated version is available online at: https://doi.org/10.1007/s40656-021-00383-w

decision making is often particularly short because experimental phase-1 patients already have advanced cancers. Even when test results are developed relatively fast, the patient may be involved in another drug trial (that prohibit further treatment) or may have become too ill to receive the experimental treatment. While some of these challenges could be overcome by developing models from biopsies or surgeries at earlier stages, it is presently unclear whether the tumours remain sufficiently stable for the models to be relevant at later stages (see also Green et al. forthcoming and Sections 3.2 and 3.3).

International publications report on similar problems and discuss various strategies to meet the temporal challenge ( $\mathrm{Xu}$ et al. 2019). These include experiments which use other mouse strains, the use of hormone supplements and different preservation media to increase engraftment rates, as well as experimental studies to determine optimal size and site for implantation (Hidalgo et al. 2014). ${ }^{9}$ Optimization of laboratory practices are also pursued through development of large-scale specialized institutions such as commercial companies offering services to research and hospitals. The Champions TumorGraft ${ }^{\circledR}$ Database in Hackensack, NJ, was in 2019 promoted as the largest database that correlate "patient-derived xenograft (PDX) model treatment response and corresponding patient clinical outcome for the same drug treatment". ${ }^{10}$ But the temporal challenge remains an obstacle even for large-scale efforts to optimize patient-derived xenografts.

Izumchenko et al. (2017) reports on the results of implementation of tumor samples from 1,163 patients at Champions Oncology. While they have achieved successful results concerning predictability of the test results in comparisons of xenograft and patient response, PDXs could be generated for only about half of the patients (578), and drug testing was conducted only for 92 of these. ${ }^{11}$ Although the authors are optimistic concerning the potential of PDXs in drug screening, they conclude that "the ability of tumors to engraft is not universal and PDX cannot be generated for every patient" (Ibid, p. 2604). Moreover, they stress that engraftment rates are highly variable across tumor types, ranging from 6 to 808 days (Izumchenko et al. 2017, Supplementary Table 1),

\footnotetext{
treatment options, ii) a performance status of 0-1, iii) a life expectancy at least 3 months, iv) normal organ function, v) measurable disease, and vi) metastatic tissue accessible for biopsy.

${ }^{9}$ Some cancers appear to grow faster if implanted orthotopically (corresponding to the original site of the tumor) compared to subcutaneous implantation. For non-small cell lung cancers the former procedure can increase the engraftment rate from $25 \%$ to $90 \%$ (see references 20, 21 cited in Hidalgo et al. 2014). This raises intriguing questions about the sensitivity of tumors to the micro and macro tumor environment (Laplane et al. 2019), and also motivates the current pursuit of PDXs as more biologically realistic models (compared to 3D cell cultures).

${ }^{10} \mathrm{https}$ ://www.championsoncology.com, accessed 14-01-2020. The specific quote can no longer be found at the webpage, as this service is no longer offered (see below for clarification).

${ }^{11}$ For these patients, treatment predictions based on PDX testing were accurate for $87 \%$.
} 
with an average time of 16 weeks. A recent news feature in Nature reports on how the company, while still creating "mouse avatars", is shifting its focus away from the personalized models, because it takes too long to achieve test results and they are too costly (Willyard 2018). Thus, the challenges described in the 1980s by Fiebig and colleagues are still hard to meet, but progress may be made through international efforts to develop "living biobanks" to speed up drug development and testing (as described below).

\subsection{PDXs as pre-clinical cancer models}

Scientific publications often explicitly distinguish the concept of a mouse avatar with the broader application of PDXs as preclinical cancer models, e.g. as translational models for biomarker identification (Terp and Ditzel 2014). Also in this context, PDXs are similarly highlighted as the ability to capture patient variation and thus account for the limitations of cell line based xenografts (CDXs). It is estimated that only about five percent of the treatments tested in cell line models end up being approved for clinical use, and PDXs are hoped to provide more clinically useful models for drug development and drug testing (Day et al. 2015; Xu et al. 2019). In the context of translational research, PDXs need not be temporally aligned to the development of specific patient cases. However, temporality is still an important feature to consider as a matter of model stability. This is illustrated in an interview with a cancer researcher in a molecular medicine lab:

$[\mathrm{P}]$ eople have started investing in PDX models where you take fresh tissue directly from the patients and transplant it into mice. They have turned out to be relatively stable, and the heterogeneity is maintained even if you passage them several times in mice. Several studies have compared gene profiles in patients with PDX models and show that there is a pretty large overlap - also over time.

The stability of PDX models over time here refers to the capacity of the model system to maintain the specific human tumor heterogeneity within the body of a living murine system. This notion of temporal stability has recently been a controversial topic of scientific debate. A key concern is that human tumors transplanted to mice undergo "mouse-specific tumor evolution" (Ben-David et al., 2017), i.e., changes in gene expression and tumor histology in the PDX compared to the human counterpart. Whereas some studies report on high degree of stability over passages (see Tables 2 
and 3 in Hidalgo et al. 2014), ${ }^{12}$ others express concerns about the observed mutational changes in mouse xenografts (Willyard 2018).

To account for the temporal challenges of scarcity of tumor material as well as model stability, researchers - quite literally - "freeze time" of the biological processes taking part in the living PDXs:

It takes quite a while before [the tumors] grow in mice. But we fortunately have good experiences with the procedure of letting them grow up to $1,2 \mathrm{~cm}$, as the law allows for, and we then cut them up in smaller pieces and put them in the freezer. Then we can have them in the freezer and take them out and grow them in new mice. (Cancer researcher in a molecular medicine lab)

Cryopreservation allows the researchers to freeze the tumor development of the samples until new mouse hosts are ready for transplantation. In this way, they can establish tumors in 15-20 mice, harvest and cryopreserve the tissue fragments when the maximally allowed size is reached, and retransplant smaller tumor pieces in new mice hosts when needed. The procedure allows researchers to expand on the amount of tumor tissue available while developing maximally identical PDXs for comparative studies or future drug testing. It is worth noting that the concept of a PDX here refers not only to the whole xenograft system, but to the characteristics of a specific human tumor that can retain its identity through cryopreservation and passaging in different mice. Hence, the mice become generic proxies (Sharp 2014, p. 46), in the sense that several mice become vehicles for the development of the personalized models.

\subsection{Extending translational and collaborative spaces}

Cryopreservation of PDXs also makes possible the establishment of so-called living biobanks where researchers can collectively contribute and share viable tissue cultures (Lisanti and Tanowitz 2012). By working together to create a library of different PDXs, researchers can get easier access to the specific cancer models needed at a specific time. Thus, the value of PDXs should not only be understood through their epistemic virtues (representation of specific cases) but also through their collaborative potential to enable or speed up research processes (cf. Michael et al. 2005; Davies

\footnotetext{
${ }^{12}$ Some studies document stability up to 10 passages (see 25 and 26 in Hidalgo et al. 2014).
} 
2012). The collaborative potential of PDXs is illustrated in the following quote with the previously mentioned cancer researcher:

Through [cryopreservation and passaging] we try to expand on the amount of tissue available. But we also use PDXs for collaborative purposes. We have for instance collaborated with [researchers] in Singapore who needed a PDX-model, and that study is now published. We also have a collaboration with some people in Prague, and now also with some in the Netherlands. Although we are not currently part of consortiums, we use the PDXs to establish our own collaborations. This is possible, in part, because not that many work on this yet, and I also don't think the availability of tissue material is equally accessible in all countries.

As the call for more diverse cancer models intensify, there is an increasing need to make bodily material as well as knowledge circulate across laboratories and even across national boundaries. The last sentence of the quote also illustrates how scarcity of tissue (due to constraints on patient availability or time) not only presents a limiting challenge but also productively can open new collaborative spaces (cf. Netz 2004). The researcher clarifies that sometimes the cryopreserved PDXs are shipped to the collaborators and grown in new mice for experimental purposes. Another time the researcher travelled with a frozen sample and established it in an animal laboratory abroad. Hence, making data and biological samples travel in space and time depends also on experimental expertise, regulatory constraints, and collaborative relations.

The use of PDXs as preclinical models potentially reshapes institutional boundaries by shortening the distance between laboratory research and clinical practice, as well as between academic research and pharmaceutical companies. For instance, PDXs are used as models for testing of new cancer treatments in so-called "mouse clinical trials", where academic researchers collaborate with pharmaceutical companies to explore how different tumor types react to the same treatment (see e.g., Koopman et al. 2019). Another highlighted opportunity of PDXs is the establishment of so-called co-clinical trials. The term initially referred to the use of Genetically Engineered Mouse Models (GEMMs) as experimental models developed in parallel to clinical trials with the purpose of identifying mechanisms underlying development of treatment resistance (Hidalgo et al. 2014), but PDXs are now explored for similar purposes (Gengenbacher et al. 2017; Clohessy et al. 2018). In the words of a cancer researcher in a molecular medicine lab: 
There are plans to make co-clinical trials where you have patients receiving treatments and where you make the same treatments on a panel of mice. As it is now, clinical trials are set up in very strict ways with high constraints on what you can and cannot do. Maybe PDXs can make it possible to take tissue samples more often and measure these while you give the treatments. In this way, you can have more tissues and more detailed investigations of a given response.

PDXs are here envisioned to serve as an experimentally accessible substitute of patients that participate in clinical trials in real-time. Since the regulatory constraints are different for patientderived murine models, and since PDXs can expand the amount of tissue material, co-clinical trials allow for drug testing on preclinical models that are as close to the target patients as possible. The researcher highlights that PDXs have allowed them to establish collaborations with pharmaceutical companies that have more resources for conducting trials at larger scales.

A recent review in Nature Clinical Oncology reports on attempts to combine the role of patient-specific drug testing and preclinical models through what they refer to as mouse hospitals, i.e., co-clinical trials on PDXs conducted in hospital laboratories, parallel to a clinical trial with patients who have donated tumor samples to the PDXs (Gengenbacher et al. 2017). In this setup, patients do not wait for test results from the PDX to receive treatment like the previous examples described in Sections 2 (mouse avatars) and 3.1 (Fiebig's studies). Instead, the vision is to develop PDXs that are temporally ahead of the clinic in a treatment series - and thus in a position to show the way for the patient treatment. For example, it may be possible to test the efficacy of several alternative treatments in parallel to a clinical patient trajectory, which may allow clinicians to be better prepared for decision making in case the patient's cancer develops resistance to one type of treatment. PDXs may thus contribute to reshape the institutional boundaries of clinical trials and potentially speed up translation to a point where there ideally is no gap between research and clinic. Yet, the vision of "instant translation" is preconditioned by the availability of PDXs to match patient tumors at the right time. To overcome the temporal challenge, multiple researchers point to the need to move beyond the ideal of fully individualized mouse avatars to develop living biobanks where type-specific specimens can be stored, cryopreserved and made available for future reuse in research or the clinic. 
Living biobanks are currently emerging to provide a research infrastructure for non-profit cancer centers and universities. For example, the EU funded consortium EurOPDX was launched in 2013 to "build large collections of models to cover cancer heterogeneity" 13 via a data repository and a tissue biobank consisting of cryopreserved PDX-based tumor samples. Another example is PDXFinder, a global cancer repository supported by the US National Institutes of Health and the National Cancer Institute, and which in 2019 contained 2888 PDX models (Conte et al. 2019). ${ }^{14}$ Such repositories are hoped to also allow for clinical applications within personalized medicine:

For patients whose tumors do not take in mice or those who require a long time to be established and characterized, an alternative to the Avatar strategy could be to orient treatment choice based on drug response of a similar PDX. Biopsies of primary tumors or metastases would be molecularly characterized and compared with available PDX collections from the same pathology, for which responses to chemotherapies and targeted agents have been previously determined. (Hidalgo et al. 2014, p. 1008).

The potentials of such repositories for personalized treatment are currently debated alongside investigations of the significance and degree of patient-specific heterogeneity. Such initiatives illustrate the potential role of consortia in managing scarcity of data and biological material through collective storing of research resources (Leonelli and Ankeny 2012; Pinel 2020). Similar to how scientists currently cryopreserve samples from endangered species, PDXs from patients with specific and often rare genetic variants can be stored for later reuse.

In summary, development of new experimental models not only have epistemic implications for relations between experimental animal and human patient. They also reconfigure regulative, social, and institutional boundaries. PDXs illustrate this point by their way of facilitating connections between academic collaborators and pharmaceutical companies, and between research and clinic. Patient-stratification based on molecular profiling greatly narrows the patient population available for enrollment in trials, and PDXs are often highlighted as a way to overcome the very need for testing on large panels of mice or human patients (Gengenbacher et al. 2017). ${ }^{15}$ In other

\footnotetext{
${ }^{13}$ www.europdx.eu, accessed March 132020.

${ }^{14} \mathrm{http}$ ://www.pdxfinder.org/about/, accessed March 13 2020. An important part of the work of the consortium is also the development of standards for laboratory practices and information sharing (see also Leonelli and Ankeny 2012).

${ }^{15}$ Hence, in these new translational spaces, patient-derived models also have an interesting potential to change existing regulatory practices concerning the gold standards of animal-based testing procedures. Discussing these aspects is, however, beyond the scope of this paper.
} 
words, the model virtues of standardization and comparability are traded for the virtue of a better representational match. However, temporal constraints remain a complicating factor, which creates a market for exchange of PDXs developed from patients with similar tumor profiles. As we shall see in the following, the temporal challenge also makes researchers explore different model systems that balance model virtues and constraints in different ways.

\section{Towards more or less humanized animal models?}

PDXs are highlighted as models embodying tumors with "the greatest similarity to human disease yet achieved" (Day et al. 2015, p. 42). Compared to in vitro cell cultures, PDXs better account for the biological context of the tumor, as the transplanted tumor is embedded in a functional metabolic system that in many ways is similar to the human body. Moreover, compared to standardized mouse models, PDXs better recapitulate the genetic variation observed in clinical context. The attempt to grow human tumors in a mouse, however, comes at the expense of the ability to study immune responses. Some have described the lack of an immune system as "the real Achilles heel" of PDX mice (Willyard 2018). The lack of immune system in PDXs makes it impossible to test immune therapies, and ignoring interactions between tumors and the immune system may weaken the ability to predict the prognosis and treatment effects. To further close the translational gap between human patients and experimental models, initiatives have been taken to "humanize" mouse models, i.e., to develop human-like immune systems in mice (Davies 2012).

The experimental procedure for producing such "humanized" mouse models is to transplant stem cells from a newborn human umbilical cord to the bone marrow of a mouse that is only a few weeks old. If the stem cells differentiate into human immune cells, the hope is that the model system will not simply reject transplanted human cancer cells but instead respond in a way similar to a human immune system. Humanized mouse models have recently been developed also from adult bone marrow stem cells, as seen in a translational model for autoimmune diseases by Dr. Megan Sykes who donated her own cells to create what she termed her "Mini-Me" (Kalscheuer et al. 2012; Perry 2013). This has sparked hope that it will be possible to develop mice models based on both immune and tumor cells from the same patient (Willyard 2018). However, researchers we have interviewed highlight persistent difficulties with this type of model, and the blurring of species boundaries is considered both a virtue and a vice. Insofar as it is possible to support human-like tumors and immune systems in mice, the translational gap between animal model and human 
patient may be smaller. So far, however, a challenge for humanized mouse models is that it is difficult to experimentally distinguish between immune responses and allogenic (rejection) responses, and it is not clear to what extent the human immune cells and connective tissues developed in mice maintain the features of human patients over time. Moreover, the attempt to develop models that maximally resemble the human patients they substitute comes with even higher tradeoffs concerning costs and temporal constraints (Day et al. 2015).

Rather than aiming for a model that maximally represents a given target, researchers often triangulate different models with complementary virtues (cf. Levins 1966; Leonelli 2007; Green 2013). This is also the case for mouse models in oncology. Traditional immunocompetent mouse models are still favored for some epistemic purposes, such as testing of some standard treatments and immune therapies. Similarly, the temporal challenge described in sections 2 and 3 have motivated some researchers to explore the possibilities of creating patient-avatars in animals that are very dissimilar to humans. Examples include initiatives to develop fly and zebrafish avatars as fast in vivo testing systems. Decades of investment in zebrafish and fruit flies as model organisms for other purposes have paved the way for creating cheaper and faster personalized models. The emphasis on rapid drug screening in precision oncology currently promotes new uses of these small model organisms (Cagan et al. 2019). ${ }^{16}$ We shall briefly examine the benefits and limitations of these below and reflect on the philosophical implications of the associated stretching of the avatar model concept.

One candidate for a much faster drug screening model is the zebrafish PDX (or zPDX) which is created by microinjection of tumor cells into zebrafish larvae (Wertman et al. 2016). A proof-of-principle study was recently published, motivated by the clinical need to identify colon cancer patients that do not respond to chemo- or radiotherapy (Costa et al. 2020). Patients with treatment-resistant tumors ( $>20 \%$ of all colon cancer patients) may not only be harmed by sideeffects of the treatments, but also by delayed initiation of other treatment strategies. The study by Costa and colleagues documents the ability of "zebrafish avatars" to distinguish between colorectal tumors that are sensitive or resistant to radio- and chemotherapy, respectively. This capacity was demonstrated for standardized cell lines as well as from rectal tumor samples from two patients. The predictive power of $\mathrm{zPDXs}$ is hard to evaluate from the small sample size, but the proof-of-

\footnotetext{
${ }^{16}$ Cagan et al. (2019) provide a useful short review of the important role of both model organisms and associated technology in research of cancer biology.
} 
This is a preprint of an article published in History and Philosophy of the Life Sciences. The final authenticated version is available online at: https://doi.org/10.1007/s40656-021-00383-w

principle study is interesting because zPDXs can provide diagnostic results within 12 days. ${ }^{17}$ The zebrafish PDXs can thus be seen as a way to opt for a different balance in the tradeoff between model virtues, i.e., between representational accuracy of cancer biology and temporal tractability of diagnostic procedures.

Another extension of the notion of 'cancer avatar' is the attempt to develop genetically personalized fruit fly models. In a recent study by Bangi, Cagan and colleagues (2019), a large panel of Drosophila melanogaster were genetically engineered to contain nine cancer-related mutations found in the tumor of a stage- 4 colon cancer patient. The genetically modified fly panel were used to test 121 FDA-approved drugs, as well as drug combinations, and the test results were submitted to a tumor board. Administration of a combination of two drugs reduced the targeted patient tumor by $45 \%$ for 11 months. ${ }^{18}$ As this study involves only one case and no control, it is difficult to compare the translational potential of fly avatars to mouse PDXs. But regardless of the specific predictive capacity of these small cancer avatars, the exploration of alternative model systems is philosophically interesting in itself, because it highlights the increasing demands for translational model systems to meet clinical time constraints.

Reduction of the spatial scale of cancer avatars, from mice to zebrafish larvae or fruit flies, allows researchers to develop xenografts directly from patient biopsies without passaging (zPDX) or to generate personalized models even without xenografting of live tumor specimens (fly avatars). The fly avatars are particularly advantageous for high-throughput combinatorial drug screens (Cagan et al. 2019). For instance, the study by Bangi et al. (2019) was based on 400,000 genetically engineered fruit flies and utilized a robotics-based screening tool to test a library of 1,500 compounds of drugs and drug combinations. The translation of results on these organisms may, however, be hampered by the size differences between human tumors and animal models. For instance, the small size and fixed genetic alterations are disadvantageous if the aim is to study larger genetic lesions, tumor dynamics, or development of tumor resistance. For this reason, the authors suggest an integrated approach where results from Drosophila studies provide inputs to organoid/PDX studies, by identifying the most promising drug candidates before investments are made in the more resource-demanding but more physiologically similar mouse avatar (Bangi et al.

\footnotetext{
17 The wet lab experiment in this case only takes 5 days. The reported implementation success rate was $43 \%$, compared to about $50 \%$ in the mouse PDXs. This aspect is, however, difficult to compare as much less material is needed to develop zPDXs and the available tissue resources are not as easily exhausted in this case.

18 After 11 months, the patient was taken off the treatment because other lesions had developed (Bangi et al. 2019). The patient died after 3 years, which the researchers estimate to be longer than he would have without this treatment (Wilson 2019).
} 
2019, p. 8). Because of the rapid development of fruit flies, having an average life cycle of only 10 days, drug testing in Drosophila could be conducted in parallel to the initial growth and passaging of mouse PDXs. ${ }^{19}$

The suggested procedure can be seen an instance of what in the philosophical literature is described as model triangulation or robustness analysis, where an ensemble of models is hoped to even out limitations associated with specific models (Levins 1966; Weisberg 2004; Leonelli 2007). The strategy of comparing diverse experimental animals have been discussed in the philosophical literature as an important way to identify conserved mechanisms and ensure robustness of model extrapolations (Logan 2002; Green et al. 2018; Parkkinen 2018). But while such discussions typically focus on synchronic representational relations between models and targets, cancer avatars exemplify how the combinatorial strategy is also motivated by the need to account for diachronic aspects of model development and data generation (cf. Leonelli 2018). In other words, the need to meet clinical demands results in the development of models that trades representational accuracy for the virtue of fast translation. Fly avatars in particular also raise the question of how dissimilar a "personalized model" can be to a human target, and whether these are better interpreted as diagnostic tools, akin to how mice and rabbits previously were used in pregnancy testing without representing this condition (Olszynko-Gryn 2014; see note 1). It is at least fair to say that fly avatars primarily represent genetic heterogeneity of tumors, rather than physiological complexity of cancer biology. This illustrates how organism choice intersects with discussions about the most salient features of cancer, and how representational accuracy is sometimes traded for temporal model virtues.

\section{Discussion and concluding remarks}

PDXs are currently promoted as preclinical models and diagnostic tools for patient-specific drug screening. By embodying tumor cells of individual patients, they are hoped to better recapitulate patient variation and thus to identify the right treatment for the right patient (Plutynski, forthcoming). In this paper we have focused on the further translation imperative to do so at the right time by examining the temporal challenges associated with aligning model development and model stability to clinical needs. We thereby hope to contribute to the discussion of how the

\footnotetext{
${ }^{19}$ While there are obvious advantages to a faster model system, the small size of fruit flies makes it impossible to transplant human cancer cells to study tumor development over time (Cagan et al. 2019, p. 318).
} 
temporalities of animal models influence their status as evidence in biomedical research and drug testing.

PDXs blur the boundary between human target and experimental organism, and between animal models and diagnostic tool. They thereby stretch or challenge the very concept of animal models of human disease. Whereas animal models, in particular model organisms, are characterized by a broad representational scope (Ankeny and Leonelli 2011), the aim of PDXs is to serve as surrogate models that stand in for specific patients. At the same time, however, patient-derived models are not straightforwardly representing individual patients (Green et al. forthcoming). Nor do they straightforwardly present a hybrid model of mouse and human. The very notion of PDX often changes depending on the context, in the clinic and in research, but also often within the same interview. Sometimes it refers to the whole xenograft system of mouse plus human tumor. In other contexts, PDX refers to the human tumor that retains the human-like identity across different passages in mouse bodies and even through cryopreservation. It is not always obvious where one body ends and another begins. Thus, PDXs are biological entities that are constructed through controlled movements of bodily material across space and time.

We have stressed that it is not only relevant to examine whether and how bodily material can be transferred from humans to animals, but also to explore the temporalities at play in model development and model stability. Species boundaries are reworked through procedures that make mice more receptive of human tissue, as seen in procedures to immunocompromise mouse models or in attempts to develop a human-like immune system in a model. At the same time, maintaining species boundaries is important to control potential temporal changes in the model system, referred to as "mouse-specific tumor evolution". The ability of PDXs to evolve in mice is thus both a vice and a virtue. It is a problem if human tumors develop murine characteristics, but evolvability in a living system is on the other hand a precondition for studies of development of treatment resistance. The aim of capturing the complexity of human tumor biology thus comes with a set of difficulties of keeping the models relatively similar and relatively stable over time, while attempting to match the temporalities of the lab and the clinic.

It could be objected that temporality is merely a practical concern of limited philosophical interest. In contrast, we view temporality as a key issue for understanding translational challenges and model choice in precision oncology, and thus of making sense of scientific practice. In discussions of what constitutes a clinically useful "cancer avatar", it is not only important to mimic the biological complexity and variation of human patients but also allow for "real-time" drug 
testing. As a result, researchers have to find ways to balance the aim of complexity of in vivo tumor biology, with the need for heterogeneous models that deliver results in a clinically relevant time window. The challenge of temporality reveals additional complexities faced in the cancer field for circulating knowledge and bodily material across boundaries. Cancer cells are often characterized by fast and uncontrolled growth and proliferation. Laboratory practices are, however, often confronted with difficulties and inertness of growing human tumors in 3D cultures or mouse hosts. Attention also to the practical difficulties thus gives a more nuanced perspective of the challenges of precision oncology.

As highlighted in Section 2, the challenge for xenograft systems to meet the temporality of the cancer clinic was described already in the 1980s by pioneers in xenograft modeling, and similar limitations are rediscovered in the current context of precision oncology. But rather than giving up the ideal of in vivo models for patient-specific drug screening, the temporal constraints currently motivate and shape new collaborative practices and exploration of alternative personalized models. Examples of the former are living PDX biobanks that not only enable circulation of knowledge but also of tissues of similar patients. Cryopreservation is here a strategy that, perhaps paradoxically, speeds up translation by "freezing time" of biological processes. The notion of a living biobank highlights how cryopreservation makes possible a temporal "zone of existence where beings are made to live and not allowed to die" (Radin and Kowal 2017, p. 6). By slowing down biological processes, cryopreservation opens for the possibility of matching extant PDXs with future patients and thus for PDXs to outlive their donor patients. In that case, specimens get a life of their own betwixt and between the worldly time of the donor and an indeterminate potentiality in a future clinic.

Similarly, we have described how the challenge to manage scarcity of tumor material and temporal constraints have opened for a potential new role of model organisms as personalized diagnostic tools. Murine systems have long been seen as the ideal organism for patient-specific drug screening due to their ability to mimic the physiological context of human tumors. But the challenge to control temporal matching of model and target currently paves the way for “personalizing” organisms which seem very far from cancer patients from a physiological perspective. Whereas PDXs and "humanized" mouse models extend the pursuit of the epistemic aim of representing as much human complexity in an experimental system as possible, tiny avatars such as zebrafish and fruit flies illustrate an attempt to trade physiological similarity for the powers of higher numbers and faster diagnostic testing. 
Although temporality has always been a practical consideration for model choice, the call for patient-specific modeling and drug screen in precision oncology exposes how temporal virtues of translational models are pushed to the center stage in response to clinical demands (see also Lowe et al. 2020). Precision oncology comes with an increasing demand for diversification of models and need for rapid patient-specific drug testing. Accordingly, the attempt to establish morphological homologies between model and target must be balanced against the need to also ensure a "temporal homology" between development of xenografted tumors and diagnostic timeframe. Cancer avatars illustrate how animals are used not only as models of human disease but also as diagnostic tools, and how surrogate models must sometime trade model virtues in order to combine these functions.

\section{Acknowledgements}

We are grateful to our informants letting us follow their practice, particularly to those who generously took the time to participate in interviews and give feedback on an earlier version of this chapter. A previous version of this paper was presented at the workshop Animal Research Unbound at University of Exeter, 2019. We would like to thank Rob Kirk, Sabina Leonelli, and Dmitriy Myelnikov for editing this topical collection and for giving constructive comments to an earlier version. Moreover, we are indebted to Simon Lohse and Robert Batterman for helpful literature suggestions and feedback. The research project Personalized Medicine in the Welfare State (MeInWe) is supported by The Carlsberg Foundation (Semper Ardens grant CF17-0016).

\section{References}

Ankeny, R. A., and Leonelli, S. (2011). What's so special about model organisms? Studies in History and Philosophy of Science Part A 42(2), 313-323.

Bangi, E., Ang, C., Smibert, P., Uzilov, A. V., Teague, A. G., Antipin, Y., ... \& Malyshev, D. (2019). A personalized platform identifies trametinib plus zoledronate for a patient with KRASmutant metastatic colorectal cancer. Science Advances, 5(5), eaav6528.

Ben-David, U., Ha, G., Tseng, Y. Y., Greenwald, N. F., Oh, C., Shih, J., ... \& Golub, T. R. (2017). Patient-derived xenografts undergo mouse-specific tumor evolution. Nature genetics, 49(11), 1567.

Bolker, J. A. (2009). Exemplary and surrogate models: Two modes of representation in biology. Perspectives in Biology and Medicine, 52(4), 485-499.

Boniolo, G. (2017). Molecular medicine: the clinical method enters the lab. What tumor heterogeneity and primary tumor culture teach us. In Nathan, M. and Boniolo, G. (eds). Philosophy 
This is a preprint of an article published in History and Philosophy of the Life Sciences. The final authenticated version is available online at: https://doi.org/10.1007/s40656-021-00383-w

of molecular medicine. Foundational issues in research and practice (pp. 23-42). New York, NY: Routledge.

Cagan, R. L., Zon, L. I., \& White, R. M. (2019). Modeling cancer with flies and fish. Developmental cell, 49(3), 317-324.

Clohessy, John G, and Pier Paolo Pandolfi. 2018. The mouse hospital and its integration in ultraprecision approaches to cancer care. Frontiers in Oncology, 8, 340. doi:10.3389/fonc.2018.00340

Conte, N., Mason, J. C., Halmagyi, C., Neuhauser, S., Mosaku, A., Yordanova, G., ... \& Meehan, T. F. (2019). PDX finder: a portal for patient-derived tumor xenograft model discovery. Nucleic Acids Research, 47(D1), D1073-D1079.

Costa, B., Ferreira, S., Póvoa, V., Cardoso, M. J., Vieira, S., Stroom, J., ... \& Greco, C. (2020). Developments in zebrafish avatars as radiotherapy sensitivity reporters - towards personalized medicine. EBioMedicine, 51, 102578.

Day, C. P., Merlino, G., \& Van Dyke, T. (2015). Preclinical mouse cancer models: a maze of opportunities and challenges. Cell, 163(1), 39-53.

Davies, G. (2012). What is a humanized mouse? Remaking the species and spaces of translational medicine. Body \& Society, 18(3-4), 126-155.

Denayer, T., Stöhr, T., \& Van Roy, M. (2014). Animal models in translational medicine: Validation and prediction. New Horizons in Translational Medicine, 2(1), 5-11.

Dietrich, M. R., Ankeny, R. A., Crowe, N., Green, S., \& Leonelli, S. (2019). How to choose your research organism. Studies in History and Philosophy of Science Part C: Studies in History and Philosophy of Biological and Biomedical Sciences, 80, 101227.

Fiebig, H. H., Schuchhardt, C., Henss, H., Fiedler, L., \& Löhr, G. W. (1984). Comparison of tumor response in nude mice and in patients. Behring Institute Mittelungen, 74, 343-352.

Fiebig, H. H., Neuman, H. A., Henss, H., Koch, H., Kaiser, D., \& Arnold, H. (1985). Development of three human small cell lung cancer models in nude mice. Recent Results in Cancer Research, 97, 77-86.

Fiebig, H. H. (1988). Comparison of tumor response in nude mice and in patients. In Winograd, B., Peckham, M., \& Pinedo, H. M. (Eds.) Human Tumour Xenografts in Anticancer Drug Development (pp. 25-30), ESO Monographs. Berlin, Heidelberg: Springer.

Flanagan, S. P. (1966). 'Nude', a new hairless gene with pleiotropic effects in the mouse. Genetics Research, 8(3), 295-309.

Harrington, J. (2013). A predicament: animal models and human tissue in medical research.

Configurations, 21(2), 183-200. 
This is a preprint of an article published in History and Philosophy of the Life Sciences. The final authenticated version is available online at: https://doi.org/10.1007/s40656-021-00383-w

Hey, S. P., \& Barsanti-Innes, B. (2016). Epistemology, ethics, and progress in precision medicine. Perspectives in Biology and Medicine, 59(3), 293-310.

Hidalgo, M., Amant, F., Biankin, A. V., Budinská, E., Byrne, A. T., Caldas, C., ... \& RomanRoman, S. (2014). Patient-derived xenograft models: an emerging platform for translational cancer research. Cancer discovery, 4(9), 998-1013.

Houchens, D. P., Ovejera, A. A., Riblet, S. M., \& Slagel, D. E. (1983). Human brain tumor xenografts in nude mice as a chemotherapy model. European Journal of Cancer and Clinical Oncology, 19(6), 799-805.

Izumchenko, E., Paz, K., Ciznadija, D., Sloma, I., Katz, A., Vasquez-Dunddel, D., ... \& Maki, R. (2017). Patient-derived xenografts effectively capture responses to oncology therapy in a heterogeneous cohort of patients with solid tumors. Annals of Oncology, 28(10), 2595-2605.

Gengenbacher, N., Singhal, M., \& Augustin, H. G. (2017). Preclinical mouse solid tumour models: status quo, challenges and perspectives. Nature Reviews Cancer, 17(12), 751.

Green, S. (2013). When one model is not enough: Combining epistemic tools in systems biology. Studies in History and Philosophy of Science Part C: Studies in History and Philosophy of Biological and Biomedical Sciences, 44(2), 170-180.

Green, S., Dietrich, M. R., Leonelli, S., \& Ankeny, R. A. (2018). 'Extreme' organisms and the problem of generalization: interpreting the Krogh principle. History and Philosophy of the Life Sciences, 40(4), 65.

Green, S., Dam, M. S. \& Svendsen, M. N. (forthcoming). Patient-derived organoids in precision oncology - towards a science of and for the individual? In Beneduce, C. and Bertolaso, M. Personalized Medicine: A Multidisciplinary Approach to Complexity. Springer International Publishing.

Kalscheuer, H., Danzl, N., Onoe, T., Faust, T., Winchester, R., Goland, R., ... \& Choi, G. (2012). A model for personalized in vivo analysis of human immune responsiveness. Science Translational Medicine, 4(125), 125ra30-125ra30.

Koopman, L. A., Terp, M. G., Zom, G. G., Janmaat, M. L., Jacobsen, K., Gresnigt-Van den Heuvel, E., ... \& Ditzel, H. (2019). Enapotamab vedotin, an AXL-specific antibody-drug conjugate, shows preclinical antitumor activity in non-small cell lung cancer. JCI Insight, 4(21), e128199.

Laplane, L., Duluc, D., Bikfalvi, A., Larmonier, N., \& Pradeu, T. (2019). Beyond the tumour microenvironment. International Journal of Cancer, 145(10), 2611-2618.

Leenaars, C. H., Kouwenaar, C., Stafleu, F. R., Bleich, A., Ritskes-Hoitinga, M., De Vries, R. B., \& Meijboom, F. L. (2019). Animal to human translation: a systematic scoping review of reported concordance rates. Journal of translational medicine, 17(1), 223. 
Leonelli, S. (2007). What is in a model? Combining theoretical and material models to develop intelligible theories. In M. D. Laubichler \& G. B. Müller (Eds.), Modeling biology. Structure, behaviors, evolution (pp. 15-35). Cambridge, MA: The MIT Press.

Leonelli, S. (2018). The time of data: timescales of data use in the life sciences. Philosophy of Science, 85(5), 741-754.

Leonelli, S., \& Ankeny, R. A. (2012). Re-thinking organisms: The impact of databases on model organism biology. Studies in History and Philosophy of Science Part C: Studies in History and Philosophy of Biological and Biomedical Sciences, 43(1), 29-36.

Levins, R. (1966). The strategy of model building in population biology. American Scientist, 54, $421-431$.

Lewis, J., Atkinson, P., Harrington, J., \& Featherstone, K. (2012). Representation and practical accomplishment in the laboratory: When is an animal model good-enough? Sociology, 47(4), 77679.

Lisanti, M. P., \& Tanowitz, H. B. (2012). Translational discoveries, personalized medicine, and living biobanks of the future. The American Journal of Pathology, 4(180), 1334-1336.

Logan, C. A. (2002). Before there were standards: The role of test animals in the production of empirical generality in physiology. Journal of the History of Biology, 35(2), 329-363.

Lowe, J. W., Leonelli, S., \& Davies, G. (2020). Training to Translate: Understanding and Informing Translational Animal Research in Pre-Clinical Pharmacology. TECNOSCIENZA: Italian Journal of Science \& Technology Studies, 10(2), 5-30.

Maugeri, P., \& Blasimme, A. (2011). Humanised models of cancer in molecular medicine: the experimental control of disanalogy. History and Philosophy of the Life sciences, 33(4), 603-621.

Malaney, P., Nicosia, S. V., \& Davé, V. (2014). One mouse, one patient paradigm: New avatars of personalized cancer therapy. Cancer letters, 344(1), 1-12.

Michael, M., S. Wainwright and Williams, C. (2005). Temporality and Prudence: On Stem Cells as “'Phronesic Things'. Configurations 13, 373-94.

Netz, R. (2004). Barbed wire: An ecology of modernity. Middletown, CT: Wesleyan University Press.

Olszynko-Gryn, J. (2014). The demand for pregnancy testing: The Aschheim-Zondek reaction, diagnostic versatility, and laboratory services in 1930s Britain. Studies in History and Philosophy of Science Part C: Studies in History and Philosophy of Biological and Biomedical Sciences, 47, 233247.

Ovejera, A. A., Houchens, D. P., \& Barker, A. D. (1978). Chemotherapy of human tumor xenografts in genetically athymic mice. Annals of Clinical \& Laboratory Science, 8(1), 50-56. 
This is a preprint of an article published in History and Philosophy of the Life Sciences. The final authenticated version is available online at: https://doi.org/10.1007/s40656-021-00383-w

Parkkinen, V.-P. (2018). Are model organisms theoretical models? Disputatio, 9(47), 471-498.

Perry, S. (2013). Mouse “avatars" advance personalized medicine. Endocrine News, Jan 2013. Available online: https://endocrinenews.endocrine.org/mouse-avatars-advance-personalizedmedicine/

Pinel, C. (2020). When more data means better results: Abundance and scarcity in research collaborations in epigenetics. Social Science Information, 59(1), 35-58.

Plutynski, A. (forthcoming). Why precision oncology is not very precise (and why this should not surprise us). In Beneduce, C. \& Bertolaso, M. (Eds.) Personalized Medicine: A Multidisciplinary Approach to Complexity. Springer International Publishing.

Rader, K. (2004). Making mice. Standardizing animals for American biomedical research, 19001955. Princeton, NJ: Princeton University Press.

Radin, J., \& Kowal, E. (2017). Introduction: The politics of low temperature. In Radin, J. \& Kowal E. (eds.), Cryopolitics: Frozen Life in a Melting World (3-25). Cambridge, MA: MIT Press.

Rygaard, J., \& Poulsen, C. O. (1969). Heterotransplantation of a human malignant tumour to "Nude" mice. Acta Pathologica Microbiologica Scandinavica, 77(4), 758-760.

Scudellari, M. (2015). My mighty mouse. The Scientist, Mar 31, 2015, Available online: https://www.the-scientist.com/cover-story/my-mighty-mouse-35712

Shanks, N., Greek, R., \& Greek, J. (2009). Are animal models predictive for humans? Philosophy, Ethics, and Humanities in Medicine, 4(1), 2.

Sharp, L. A. (2014). The transplant imaginary: Mechanical hearts, animal parts, and moral thinking in highly experimental science. Berkeley and Los Angeles: University of California Press.

Svendsen, M. N., \& Koch, L. (2013). Potentializing the research piglet in experimental neonatal research. Current Anthropology, 54(S7), S118-S128.

Svendsen, M. N., Navne, L. E., Gjødsbøl, I. M., \& Dam, M. S. (2018). A life worth living: Temporality, care, and personhood in the Danish welfare state. American Ethnologist, 45, 20-33.

Taetle, R., Honeysett, J. M., Rosen, F., \& Shoemaker, R. (1986). Use of nude mouse xenografts as preclinical drug screens: Further studies on in vitro growth of xenograft tumor colony-forming cells. Cancer, 58(9), 1969-1978.

Terp, M. G., \& Ditzel, H. J. (2014). Application of proteomics in the study of rodent models of cancer. PROTEOMICS-Clinical Applications, 8(9-10), 640-652. 
van der Worp, H. B., Howells, D. W., Sena, E. S., Porritt, M. J., Rewell, S., O’Collins, V., et al. (2010). Can animal models of disease reliably inform human studies? PLoS Medicine, 7(3), e1000245.

Wertman, J., Veinotte, C. J., Dellaire, G., \& Berman, J. N. (2016). The zebrafish xenograft platform: Evolution of a novel cancer model and preclinical screening tool. Advances in Experimental Medicine and Biology, 916, 289-314.

Weisberg, M. (2004). Robustness analysis. Philosophy of Science, 73(5), S730-42.

Willyard, C. (2018). The mice with human tumours: Growing pains for a popular cancer model. Nature news feature, 560(7717), 156.

Wilson, C. Specially created animal 'cancer avatars' could personalise treatments. NewScientist, May 22, 2019, Available online: https://www.newscientist.com/article/2204384-specially-createdanimal-cancer-avatars-could-personalise-treatments/.

Winograd, B., Peckham, M., \& Pinedo, H. M. (1988, Eds.). Human tumour xenografts in anticancer drug development. ESO Monographs. Berlin, Heidelberg: Springer.

Xu, C., Li, X., Liu, P., Li, M., \& Luo, F. (2019). Patient-derived xenograft mouse models: A high fidelity tool for individualized medicine. Oncology letters, 17(1), 3-10. 